\title{
Effects of sodium pentobarbital on symbolic matching and symbolic oddity performance
}

\author{
DAVID A. ECKERMAN \\ University of North Carolina, Chapel Hill, North Carolina 27514 \\ ROBERT N. LANSON \\ Queens College of the City University of New York, Flushing, New York 11367 \\ and \\ ROBERT BERRYMAN \\ American University, Cairo, Egypt
}

\begin{abstract}
Pigeons' conditional discrimination accuracy was equally disrupted by administering sodium pentobarbital when the discrimination required a three-alternative symbolic matching-to-sample task (if blue, select green; if green, select red; if red, select blue) as when it required a threealternative symbolic oddity-from-sample task (if blue, select anything but green; etc.). This equivalence in disruption makes difficult interpretation of prior findings showing simple matching to sample (if blue, select blue; if green, select green; etc.) to be more disrupted by drugs than was simple oddity (if blue, select anything but blue; etc.).
\end{abstract}

Two previous studies (Berryman, Cumming, Nevin, \& Jarvik, 1964; Berryman, Jarvik, \& Nevin, 1962) have shown differences in drug effects on the performance of pigeons on simple matching-to-sample and simple oddity-from-sample tasks. Specifically, $10 \mathrm{mg} / \mathrm{kg}$ of sodium pentobarbital reduced the matching accuracy from a baseline of $94 \%$ correct to $76 \%$, while the same dose only reduced oddity accuracy from $95 \%$ to $89 \%$.

In both experiments, a sample, or standard (ST) color, was presented to start a trial. This sample indicated which one of a pair of comparison colors (COs) was correct for that trial. After the subject had responded to the ST, the COs appeared; a response to one of them (the $\mathrm{CO}+$ ) was reinforced with food and a response to the other (the $\mathrm{CO}-$ ) was punished by a brief blackout. In simple matching to sample, food reinforcement was given for selecting the $\mathrm{CO}$ with the same physical characteristics as the ST; in simple oddity, for selecting any $\mathrm{CO}$ but the one with the same physical characteristics as the ST. However, as Cumming and Berryman (1965) indicate in their extensive review of

Dedicated to the memory of Dr. W.W. Cumming. His untimely death, on January 8,1970 , prevented his participation in the preparation of this report; his colleagues and co-workers thus assume full responsibility for any flaws that may detract from its merit, and hope that it will, nevertheless, show something of the excellence that characterized his work. The study was supported by Grants MH-03673 and MH-10384 from the National Institute of Mental Health, Public Health Service, to Dr. William W. Cumming. Preparation of the manuscript was partially supported by Training Grant MH-14269 from the National Institute of Mental Health, Public Health Service. Reprints are available from the first author, Department of Psychology, University of North Carolina, Chapel Hill, North Carolina 27514. these procedures, the contingency rules may specify completely arbitrary relations between STs and COs, thus defining symbolic matching and oddity. In symbolic matching, reinforcement, given a certain ST, would be given for selecting an arbitrarily assigned $\mathrm{CO}$; in symbolic oddity, for selecting any but an arbitrarily assigned $\mathrm{CO}$.

When three (or more) stimuli are available as alternative STs and COs, the simple matching and oddity cases differ in the number of $\mathrm{CO}+$ and $\mathrm{CO}$ - stimuli designated by each ST. In matching, each ST designates one $\mathrm{CO}+$ and two (or more) $\mathrm{CO}$ - stimuli. In oddity, each ST designates one $\mathrm{CO}-$ and two (or more) $\mathrm{CO}+$ stimuli. Evidence from transfer tests following extensive original training provides evidence that, for both simple matching and simple oddity, the pigeon selects the $\mathrm{CO}+$ but does not avoid the $\mathrm{CO}-$ when in the presence of a particular ST (Cumming, Berryman, \& Cohen, 1965; Berryman, Cumming, Cohen, \& Johnson, 1965). That is, the pigeon is controlled by the St rules (ST implies $\mathrm{CO}+$ ) but not by the $\mathrm{S}-$ rules (ST implies $\mathrm{CO}-$ ) in both the matching and the oddity cases. Given this analysis, matching is seen to be structurally simpler than oddity because only one $\mathrm{CO}+$ stimulus rather than two (or more) $\mathrm{CO}+$ stimuli is designated by each ST. While this account handles the more rapid acquisition of matching than oddity (Cumming \& Berryman, 1965), the greater disruption of matching by sodium pentobarbital then seems to contradict the rule of thumb that more "complex" discriminations are more easily disrupted by drugs (e.g., Dews, 1955). In the present report, we seek to provide some data to address this apparent contradiction. 
The symbolic matching and symbolic oddity cases allow a slightly different look at the issue of drug effects on matching and oddity. Here, too, only one $\mathrm{CO}+$ is designated by a ST in the symbolic matching case, while two (or more) CO+ stimuli are designated by a ST in the symbolic oddity case. The $\mathrm{CO}+$ assignments, however, differ between the simple and the symbolic versions, since they are based on identity for simple matching but are arbitrary for simple oddity and for both symbolic matching and symbolic oddity. In hope of disentangling effects of stimulus assignment from effects of complexity of S+ rule, sodium pentobarbital was given pigeons trained on symbolic matching and symbolic oddity tasks using the same stimuli, apparatus, and general procedures previously utilized in comparisons of simple matching and simple oddity by Berryman et al. $(1962,1964)$.

\section{METHOD}

\section{Subjects}

The subjects were 10 white Carneaux pigeons maintained at $80 \%$ of their free-feeding weight. They were individually housed with grit and water available ad lib. Experimental sessions were scheduled only when the bird's weight was within $\pm 15 \mathrm{~g}$ of the $80 \%$ value. Food reinforcements and supplemental feedings in the home cage were a mixture of $50 \%$ vetch, $40 \%$ Kaffir corn, and $10 \%$ hemp seed.

\section{Apparatus}

The experimental chamber (Cumming \& Berryman, 1965) had three translucent response keys and a grain magazine mounted on one wall of a triangular compartment. The keys could be individually transilluminated with blue, green, or red Christmas-tree bulbs. Ceiling lights provided general illumination. Automatic control and recording apparatus was housed remotely in a separate room.

\section{Procedure}

In preliminary training all birds were trained to eat from the grain magazine and then shaped to peck any of the three keys when they were transilluminated with any one of the three colors.

Five birds then received 47 training sessions, each of 140 trials, on the symbolic matching task as specified in Table 1, that is, blue ST, peck green; green ST, peck red; red ST, peck blue. The remaining five subjects received 50 training sessions, also of 140 trials, on the symbolic oddity task, that is, blue ST, peck blue or red; green ST, peck green or blue; red ST, peck red or green.

The procedure was as described by Cumming and Berryman (1965). For both tasks, responding to $\mathrm{CO}+$ gave $3 \mathrm{sec}$ grain access; responding to $\mathrm{CO}-$ produced a 3-sec blackout, during which all illumination in the chamber was turned off. Following either blackout or reinforcement, a 30-sec intertrial interval ensued, during which all keys were dark and all responses without effect. At the start of a trial, one ST was presented on the center key. A single peck to it illuminated the two CO keys. Since a given $\mathrm{CO}+$ appeared an equal number of times on the left and on the right keys, there were 12 trial types, rather than the 6 shown in the schematic representation of Table 1.

\section{Assessment of Drug Effects}

Sodium pentobarbital dose levels of 7.5 and $12.5 \mathrm{mg} / \mathrm{kg}$ were used with both procedures. The drug was administered via the IM route $10 \mathrm{~min}$ before experimental sessions. For one bird
(234) who did not complete a session (no responses within $45 \mathrm{~min}$ ) with $12.5 \mathrm{mg} / \mathrm{kg}$, a $10.0-\mathrm{mg} / \mathrm{kg}$ dose was substituted as the highest value. Sessions were given every other day; hence, 4 days intervened between successive drug administrations.

\section{RESULTS}

Both the symbolic matching and symbolic oddity performances showed a decrement in accuracy with 7.5 - and $12.5-\mathrm{mg} / \mathrm{kg}$ (or $10.0-\mathrm{mg} / \mathrm{kg}$ ) administrations of sodium pentobarbital (see Figure 1). The drop with the $7.5-\mathrm{mg} / \mathrm{kg}$ dose was less than that with the larger dose for all subjects. Accuracy had substantially recovered toward nondrug levels, however, by the end of drug sessions at either dose (exception: Bird 235 at the $12.5-\mathrm{mg} / \mathrm{kg}$ dose).

No clear difference was apparent between the effect of sodium pentobarbital on symbolic matching and on symbolic oddity performances (from a multivariate ANOVAR across successive determinations or across successive sixths of sessions, no significant effect across conditions was found).

For both symbolic matching and symbolic oddity

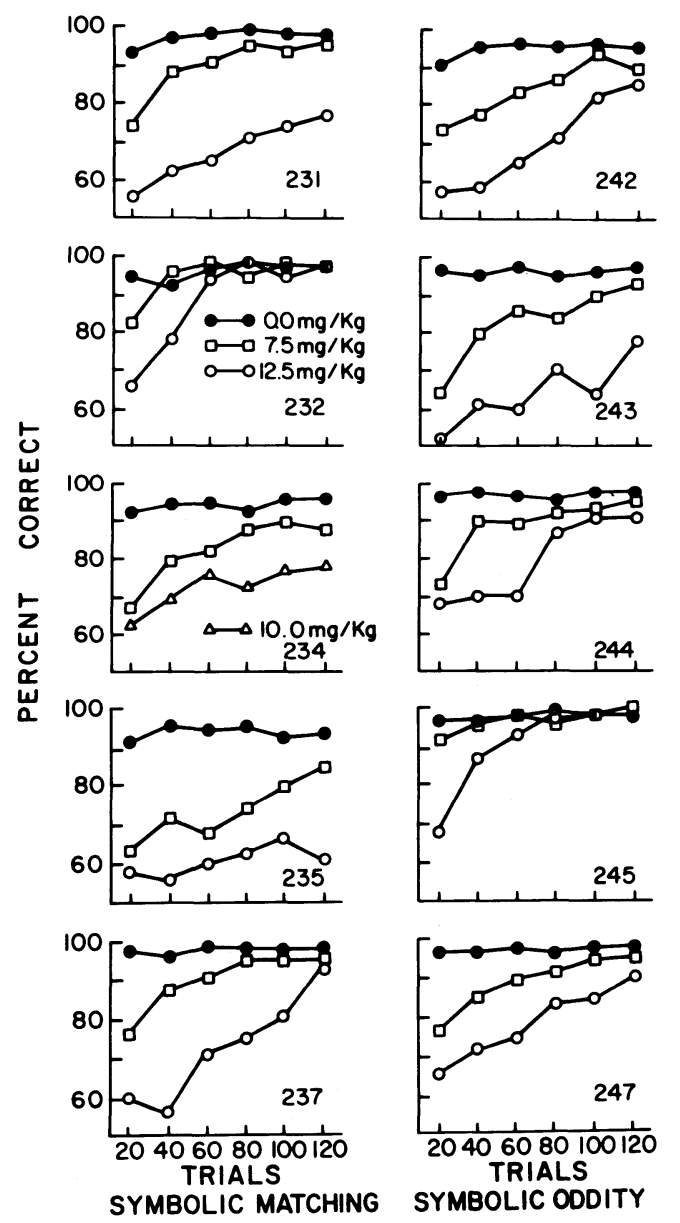

Figure 1. Accuracy changes during drug and control sessions for symbolic matching and symbolic oddity subjects. Bird numbers are indicated for each panel. 
Table 1

Trial Types and Reinforcement Designations for Simple and Symbolic Matching and Oddity for Blue (B), Green (G), and Red (R) Stimuli Appearing Both as STs and COs

\begin{tabular}{ccccccccc}
\hline & \multicolumn{4}{c}{ Simple } & \multicolumn{4}{c}{ Symbolic } \\
Trial & \multicolumn{3}{c}{ Matching } & \multicolumn{2}{c}{ Oddity } & \multicolumn{2}{c}{ Matching } & Oddity \\
Type & ST & COs & ST & COs & ST & COs & ST & COs \\
\hline 1 & B & B* G & B & B G* & B & G* B & B & G B* \\
2 & B & B* R & B & B R* & B & G* R & B & G R* \\
3 & G & G* B & G & G B* & G & R* G & G & R G* \\
4 & G & G* R & G & G R* & G & R* B $^{*}$ & G & R B* \\
5 & R & R $^{*}$ B & R & R B* & R & B* R & R & B R* \\
6 & R & R $^{*}$ G & R & R G* & R & B* G & R & B G* \\
\hline
\end{tabular}

Note-Correct $\mathrm{CO}$ s are indicated by an asterisk. For further explanation, see text.

subjects, the decreased accuracy was usually accompanied by an increased proportion of pecks to one of the side-key locations (the right side for some and the left side for other subjects). At the $12.5-\mathrm{mg} / \mathrm{kg}$ dose, a median of $60 \%$ of responses was made to the preferred side (20\% position preference).

Consideration of the stimulus configuration in symbolic matching and oddity suggests a further analysis. Inspection of Table 1 shows that half the configurations in both symbolic conditions are trials in which three different colors are present simultaneously. These three-color trials do not occur under simple matching or oddity rules. Furthermore, the remaining two-color trials (Types 1, 3, and 5 in Table 1) in symbolic matching have the same configuration and reinforcement contingency as simple oddity trials. For example, in the symbolic matching procedure, when the ST is blue, the $\mathrm{CO}+$ is designated as green. With this ST, the COon half the trials will be blue. Such a configuration appears during simple oddity-from-sample training. Similarly, the same two-color trial types in the sym- bolic oddity procedure can be shown to have their counterparts in simple matching. One can compare the accuracy on the two- and three-color trials of both symbolic procedures to determine whether configurations differing from those found in the simple matching or oddity rule are subject to differential drug effects.

Table 2 shows the separate accuracies for all birds over the three dose levels. Within birds, at a given dose, no notable difference in accuracy can be seen between the two- and three-color trials. A further comparison can be made across procedures for a given drug dose to see whether the differential sensitivity of matching and oddity to pentobarbital, as demonstrated in earlier studies, was in evidence. The prediction from the previous research would be that the two-color symbolic oddity trials (those equivalent to simple matching) would be more affected by the drug than the two-color symbolic matching trials (those equivalent to simple oddity). Although a comparison of means at a given dose suggests a trend in the predicted direction, the ranges of variability show sufficient overlap to rule out statistical significance.

\section{DISCUSSION}

As in prior work (Berryman et al., 1962, 1964), sodium pentobarbital reduced accuracy of pigeons' conditional discrimination performance. While oddity performance was previously found to be less affected by the drug than was matching performance, however, symbolic oddity and symbolic matching performances were about equally affected. Because apparatus and general procedures in the present work were the same as those in the prior studies, a close comparison of accuracy reduction may be made. Despite the difference in doses used (7.5 and $12.5 \mathrm{mg} / \mathrm{kg}$ rather than 5.0 and $10.0 \mathrm{mg} / \mathrm{kg}$ ), comparison shows accuracies for the symbolic discriminations were reduced more than were accuracies for simple oddity and to about the same degree as were accuracies for simple matching.

Unpublished transfer data from the present subjects (yellow

Table 2

Accuracies for Two- and Three-Color Trials in Symbolic Matching and Oddity for Drug and Control Conditions

\begin{tabular}{|c|c|c|c|c|c|c|c|c|c|}
\hline \multirow[b]{2}{*}{ Bird } & \multicolumn{3}{|c|}{ All Trials } & \multicolumn{3}{|c|}{ Two-Color Trials } & \multicolumn{3}{|c|}{ Three-Color Trials } \\
\hline & 0.0 & 7.5 & 12.5 & 0.0 & 7.5 & 12.5 & 0.0 & 7.5 & 12.5 \\
\hline & \multicolumn{9}{|c|}{ Symbolic Matching } \\
\hline $\begin{array}{l}231 \\
232 \\
234^{*} \\
235 \\
237\end{array}$ & $\begin{array}{l}96.6 \\
95.8 \\
94.1 \\
93.1 \\
97.7\end{array}$ & $\begin{array}{l}88.9 \\
94.3 \\
82.3 \\
73.5 \\
90.0\end{array}$ & $\begin{array}{l}67.7 \\
88.0 \\
72.3 \\
60.8 \\
72.1\end{array}$ & $\begin{array}{l}95.7 \\
96.2 \\
93.8 \\
98.2 \\
98.1\end{array}$ & $\begin{array}{l}89.2 \\
93.7 \\
84.3 \\
72.0 \\
90.0\end{array}$ & $\begin{array}{l}69.1 \\
88.0 \\
73.0 \\
61.0 \\
73.7\end{array}$ & $\begin{array}{l}97.2 \\
94.1 \\
94.3 \\
89.7 \\
97.4\end{array}$ & $\begin{array}{l}88.6 \\
95.0 \\
80.0 \\
73.7 \\
90.0\end{array}$ & $\begin{array}{l}66.1 \\
88.0 \\
71.7 \\
60.7 \\
70.4\end{array}$ \\
\hline $\begin{array}{l}\text { Mean } \\
\text { SD }\end{array}$ & $\begin{array}{r}95.5 \\
3.1\end{array}$ & $\begin{array}{r}85.8 \\
6.2\end{array}$ & $\begin{array}{r}72.2 \\
7.7\end{array}$ & 96.4 & 85.8 & 73.0 & 94.5 & 85.5 & 71.3 \\
\hline $\begin{array}{l}242 \\
243 \\
244 \\
245 \\
247\end{array}$ & $\begin{array}{l}95.1 \\
96.5 \\
97.0 \\
97.8 \\
99.3\end{array}$ & $\begin{array}{l}84.5 \\
82.1 \\
87.2 \\
96.6 \\
92.0\end{array}$ & $\begin{array}{l}70.2 \\
63.8 \\
79.5 \\
91.8 \\
85.8\end{array}$ & $\begin{array}{r}\text { Sy } \\
96.7 \\
97.6 \\
97.2 \\
98.7 \\
99.8\end{array}$ & $\begin{array}{c}\text { lic Od } \\
84.9 \\
82.9 \\
84.9 \\
97.6 \\
92.2\end{array}$ & $\begin{array}{l}71.3 \\
67.6 \\
81.9 \\
93.6 \\
86.9\end{array}$ & $\begin{array}{l}93.2 \\
95.2 \\
95.9 \\
96.7 \\
98.9\end{array}$ & $\begin{array}{l}83.9 \\
81.3 \\
89.2 \\
95.6 \\
91.6\end{array}$ & $\begin{array}{l}68.9 \\
59.9 \\
76.9 \\
89.9 \\
84.6\end{array}$ \\
\hline $\begin{array}{l}\text { Mean } \\
\text { SD }\end{array}$ & $\begin{array}{r}97.1 \\
1.8\end{array}$ & $\begin{array}{r}88.5 \\
5.9\end{array}$ & $\begin{array}{r}78.2 \\
7.3\end{array}$ & 98.0 & 88.5 & 80.3 & 96.0 & 88.3 & 76.0 \\
\hline
\end{tabular}

*Bird 234 was given 10.0 rather than $12.5 \mathrm{mg} / \mathrm{kg}$. These points are not included in the column mean. 
bulbs were substituted for blue bulbs and training was continued) showed that $\mathrm{S}+$ rules again characterized the symbolic matching and symbolic oddity performances and that $S$ - rules did not. That is, when yellow was a new CO+ for an old ST, performance deteriorated. When yellow was a new $\mathrm{CO}-$ for an old ST, performance was relatively unaffected. It might be noted that when yellow was an ST, results were in accord with the coding hypothesis as described by Cumming et al. (1965).

The widely held view that more "complex" discriminations may be more readily disrupted by drugs than "simpler" discriminations (Dews, 1955) suggests the possibility of analyzing performance in terms of complexity of $S+$ rules. One may assume that the number of $\mathrm{CO}+$ stimuli per ST is a measure of complexity. In the present study, since performance generated by the symbolic conditional discriminations may be characterized by $\mathrm{S}+$ rules (just as prior work has shown for simple matching and oddity), then symbolic matching (one $\mathrm{CO}+$ per ST) may be described as simpler than symbolic oddity (two $\mathrm{CO}+$ per ST). The present data do not support this view regarding complexity and drug effect, since performance on the two symbolic tasks was equally affected. The earlier findings of the insensitivity of simple oddity (two $\mathrm{CO}+$ per ST) relative to simple matching (one $\mathrm{CO}+$ per ST) also contradict the suggestion of complexity as the critical variable.

Another possible dimension of complexity is the number of stimuli presented on a given trial. Detailed analysis of the twocolor and three-color trials, however, showed no differential drug effects. These failures to demonstrate "complexity" as a controlling variable lead us to agree with Dews that it would be preferable to describe the differential sensitivities to drugs, where found, in terms of the operational difference between the procedures. We cannot reject the possibility, however, that complexity could be defined by some other, yet unknown, stimulus dimension.

A second possible determinant of the differential disruption of simple matching and oddity by drugs might be in the stimulus configuration per se. Because the two-color matching trials in symbolic matching are identical to simple oddity trials and because the two-color trials in symbolic oddity are like simple matching trials, if the drug effect were a function of stimulus configuration, we would expect symbolic matching to be relatively immune to disruption. The failure to find a difference between the two symbolic tasks suggests that configuration is not the critical determinant.

The present data thus eliminate two proposals to account for the relative insensitivity of simple oddity to drug disruption, even though they do not offer support for any known account of the difference.

\section{REFERENCES}

Berryman, R., Cumming, W. W., Cohen, L. R., \& Johnson, D. F. Acquisition and transfer of simultaneous oddity. Psychological Reports, 1965, 17, 767-775.

Berryman, R., Cumming, W. W., Nevin, J. A., \& Jarvik, M. E. Effects of sodium pentobarbital on complex operant discrimination. Psychopharmacologia, 1964, 6, 388-398.

Berryman, R., Jarvik, M. E., \& NeVIN, J. A. Effects of pentobarbital, lysergic acid diethylamide, and chlorpromazine on matching behavior in the pigeon. Psychopharmacologia, 1962, 3, 60-65.

Cumming, W. W., \& Berryman, R. The complex discriminated operant. In D. Mostofsky (Ed.), Stimulus generalization. Stanford, Calif: Stanford University Press, 1965. Pp. 284-330.

Cumming, W. W., Berryman, R., \& Cohen, L. R. Acquisition and transfer of zero-delay matching. Psychological Reports, 1965, 17, 435-445.

Dews, P. B. Studies on behavior: II. The effects of pentobarbital, methamphetamine and scopolamine on performances in pigeons involving discriminations. Journal of Pharmacology and Experimental Therapeutics, 1955, 115, 380-389.

(Received for publication November 15, 1977.) 